\title{
EXPERIMENTAL EVALUATION OF MULTIMODAL HUMAN COMPUTER INTERFACE FOR TACTICAL AUDIO APPLICATIONS
}

\author{
Zeljko Obrenovic, Dusan Starcevic \\ University of Belgrade, Belgrade, Yugoslavia \\ obren@computer.org, starcev@fon.bg.ac.yu \\ Emil Jovanov \\ University of Alabama in Huntsville, Huntsville, Alabama
}

\begin{abstract}
Mission critical and information overwhelming applications require careful design of human computer interface. Typical applications include night vision or low visibility mission navigation, guidance through a hostile territory, and flight navigation and orientation. Additional channels of situation critical information can be better perceived using simultaneous audio and visual presentation. In this paper we present a multimodal simulation system as an open development environment for evaluation of sonification and visualization paradigms in augmented reality systems. The environment is implemented as a virtual audio-visual scene using Java3D package, and used for performance measurements in tactical guidance applications. We present the results of target tracking experiment with 10 participants using visual, acoustic and combined guidance. The experiment has shown that acoustic presentation significantly improve the quality of human-machine interaction and reduces error during guidance tasks.
\end{abstract}

\section{INTRODUCTION}

Exploration of perceptual features of sonification paradigms requires a flexible environment that could be easily customized to suit user or applications needs. We believe that tactical audio could be efficiently used as a feedback for achieving a goal, and resolve some problems in augmented reality systems. In addition to displaying spatial information using spatialized audio, we can use elements of audio perception to convey situational awareness. However, wider acceptance of sonification and tactical audio applications will primarily depend on quality of user interfaces and how fast users can effectively learn to use the environment. Evaluation of user learning curves is vital to continuing improvement of tactical sonification.

A computer generated sonic scene leaves the visual field unimpaired, unobstructed, and ready for investigation of the environment for surprises. This is very important in a range of the mission critical applications, such as surgical navigation, aircraft navigation and safety, spacecraft docking, night vision mission navigation, and flight navigation and orientation [1-4].

We have investigated psychological features of different sonification paradigms in customized sonification environments. In this paper we present our experience and preliminary performance measures in a multimodal simulation and training system for tactical audio applications. Typical issues in sonification and tactical audio applications are given in chapter two. Implementation of the environment is presented in chapter three. An experimental evaluation of user performance during target tracking task with combined visual and acoustic feedback is given in the same chapter. Chapter four concludes the paper.

\section{MULTIMODAL HUMAN-COMPUTER INTERFACES}

Multimodal systems process multiple natural input modes in a coordinated manner with multimedia or multimodal system output. Multimodal systems represent a paradigm shift away from conventional windows-icons-menus-pointers (WIMP) paradigm. Well-designed multimodal systems integrate complementary modalities to yield a highly synergistic mix in which the strengths of each mode are capitalized upon and used to overcome weaknesses in the other. Such systems can function more robustly than unimodal systems $[1,2,5-7]$.

\subsection{Sonification}

After visualization, sonification is the second most important presentation modality $[3,4]$. Multimodal human-computer interface design is a complex design problem due to the nature of the cognitive information processing [6]. The most important advantages of acoustic data presentation include faster processing than visual presentation, sound is easier to focus and localize attention in space, and good temporal resolution (almost an order of magnitude better than visual). Therefore, sonification could be used to present additional information channels, releasing visual sense for other tasks.

Disadvantages of acoustic data presentation include: difficult perception of precise quantities and absolute values, limited spatial resolution, interdependence of some sound parameters (for example pitch depends on loudness), interference with other sound sources like speech, absence of persistence, and individual user perception dependency.

It could be seen that some characteristics of visual and acoustic perception are complementary. Therefore, sonification naturally extends visualization. The system that supports sonification must provide the ability to extract the relevant information features. The most important sound characteristics affected by sonification are: Pitch, as the subjective perception of frequency, Timbre as characteristic of instrument generating 
sounds, Loudness or subjective volume, and Location of sound source.

In our previous research, we have applied sonification of brain electrical activity to facilitate insight into complex spatiotemporal patterns of brain electrical activity $[5,6]$.

\subsection{Tactical Audio}

Tactical audio uses audio feedback to facilitate the precise and accurate positioning of an object with respect to another object. This has valuable application in computer assisted surgery. Just as musicians use aural feedback to position their hands, surgeons could position instruments according to a pre-planned trajectory, pre-placed tags or cues, or anatomical models [7]. In the course of the typical diagnostic surgical procedure there are numerous needle placement errors, especially with regard to insertion depth, e.g., missing the tumor in a biopsy. Although ultrasound and other imaging modalities attempt to alleviate this problem, the nature and configuration of the equipment requires the surgeon to take his/her eyes off the patient. The use of tactical audio feedback enables the surgeon to effect a precise placement by enhancing his/her comprehension of the three-dimensional position of a surgical implement with respect to some predetermined desired position within the patient's body. In addition to enabling more precise placement of surgical instruments, and therefore improved surgical outcomes, we believe this approach will yield substantial savings in time and cost. This clearly benefit both patient and healthcare provider [7].

Tactical audio system development critically depends on optimally intuitive sonification paradigm. Therefore, we are working on tactical audio development system that can allow testing and quantitative measurements of different solutions [8].

\section{A MULTIMODAL SIMULATION SYSTEM}

We developed a multimodal simulation and training environment in order to quantitative evaluate the effectiveness of sonification methods and asses learning curve in a large population of users [8]. The environment is based on commercially available technology, implemented using Java3D [9] and standard Java components. Java3D is primarily used for acoustic 3D effects. This approach allows transparent execution on stand-alone workstations, as well as in a distributed Web environment in the form of Java applet.

The environment consists of an interaction space and of a control interface, as presented in Figure 1. Main interaction between a user and the environment occurs in the interaction space, where the user tracks animated target. In the control interface the user can set various parameters including multimodal combination, speed, and file parameters (Figure 2).

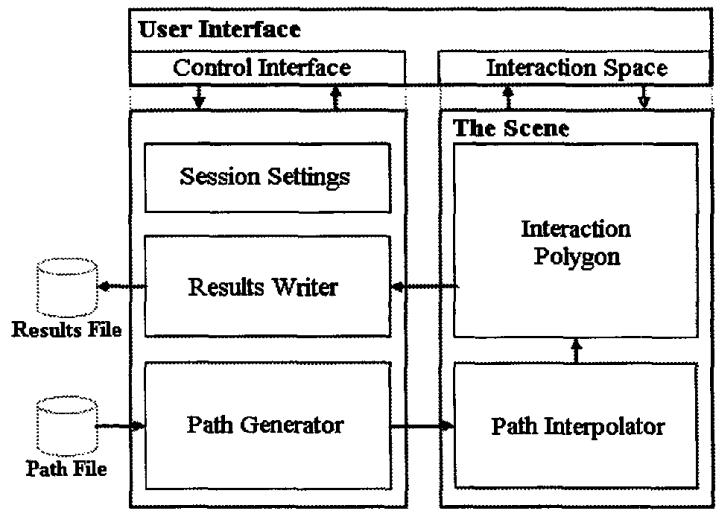

Figure 1. Architecture of the tactical audio development environment.

The environment is used to estimate the possible performance improvement of tactical audio system. However, the environment is designed flexible in order to allow easy plugand-play addition of new graphical and acoustic modes.

A typical user interface of the multimodal tracking environment is presented in Figure 2. Interaction space of the scene contains:

- target object,

- trajectory control object

- user-controlled cursor object, and

- sonification objects (sound objects).

The trajectory of target object is controlled either from a random generated set of coordinates or from a file. In the current version of the environment, cursor object is mouse-controlled. User simply tries to "cover" top of the target using cursor. A special object that contains a timer and a position interpolator controls dynamics of target object. Position interpolator calculates new target position on each timer tick using discrete set of coordinates to provide "smooth" trajectory in space/time.

Java3D allows use of sound objects that could be manipulated in real-time. Objects are defined as sound files, and their parameters (such as position, intensity, etc.) are manipulated directly. We experimented with two different sound objects:

- variable position sound object representing target position in space; and

- variable intensity sound object representing a distance of the cursor from the target.

Users can customize their own mappings of target position to sound position, and target distance to sound intensity. 


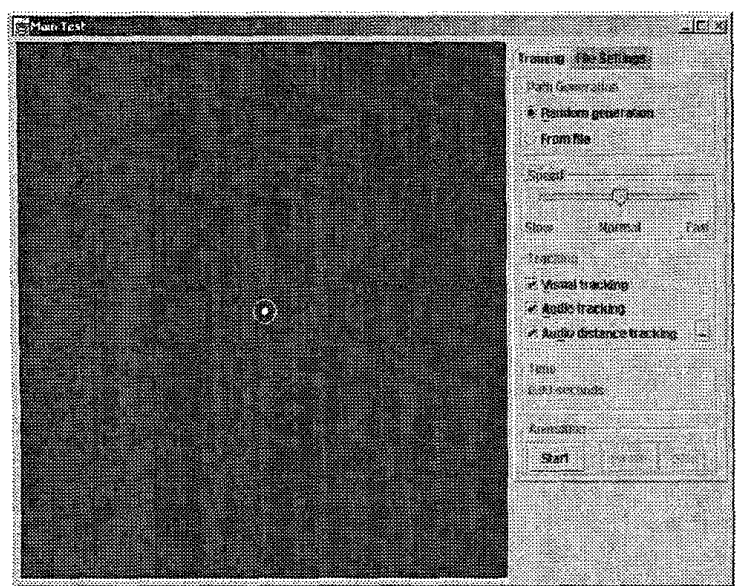

Figure 2. User interface of the multimodal target tracking development environment.

\subsection{Experiment}

In the target tracking experiment we assess user performance in two-dimensional object-tracking applications. Target object was positioned according to predefined trajectory taken from a file, and coordinates of both target and the cursor object were written to the result file for off-line processing. In addition to graphical presentation mode, we generated two modes of acoustic feedback: positional - based on stereo effect, in which target position determines sound object position in 3D world [6], and distance, where the intensity of the sound object depends on relative distance from the target [8]. With these three modes we created four multimodal combinations (MMCs) (see Table 1).

Table 1. Multimodal combinations (MMCs) used in the target tracking experiment.

\begin{tabular}{|c|c|c|c|}
\cline { 2 - 4 } \multicolumn{1}{c|}{} & $\begin{array}{c}\text { Graphical } \\
\text { mode }\end{array}$ & $\begin{array}{c}\text { Positional } \\
\text { sound }\end{array}$ & $\begin{array}{c}\text { Distance } \\
\text { sound }\end{array}$ \\
\hline $\mathrm{MMC1}$ & + & & \\
\hline $\mathrm{MMC2}$ & + & + & \\
\hline $\mathrm{MMC} 3$ & + & & + \\
\hline $\mathrm{MMC} 4$ & + & + & + \\
\hline
\end{tabular}

The experiment included 16 tests for each subject. Each multimodal combination was tested with four target speeds, as represented in Table 2.

Each MMC lasted for 35 seconds. The experiment started with lowest speed, where all combinations were tested. After that the target speed was increased. Before transition to the higher speed, a subject could take a break that lasted 30 seconds. Total duration of the experiment for each subject was around 10 minutes.
Table 2. Average speeds of the target object in the target tracking experiment.

\begin{tabular}{|c|c|}
\hline Speed ID & $\begin{array}{c}\text { Average speed } \\
\text { (pixels per second) }\end{array}$ \\
\hline 1 & 19 \\
\hline 2 & 75 \\
\hline 3 & 128 \\
\hline 4 & 226 \\
\hline
\end{tabular}

This experiment ran on a $433 \mathrm{MHz}$ Celeron PC with an SiS 6326 graphics card and Crystal PnP MPU-401 sound card in Windows Millennium operating system. The frame rate was about 20 frames per second.

\subsubsection{Subjects}

Ten subjects participated in the study. All of them had normal or corrected-to-normal vision and normal hearing. Age of the participants ranged from 19-35 years.

\subsection{Results}

The main performance parameter of our experiment and data analysis was tracking error. The tracking error is a distance between user's cursor and the position of the target object. Smaller error represents better result.

Averaged positioning exrors of all ten participants are shown in Figure 3. This figure illustrates dependencies among speed, multimodal combination and average error.

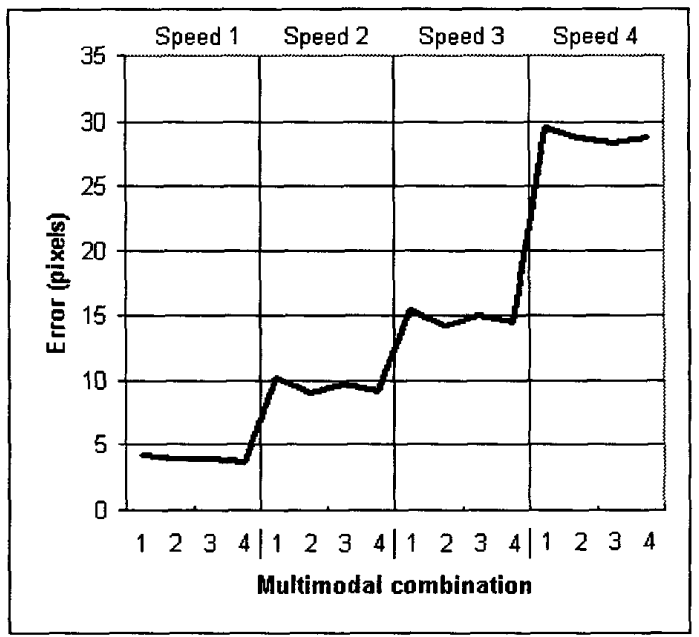

Figure 3. Dependencies among average error, speed, and multimodal combination. 
As expected, higher target speed generates larger average error. It is interesting to note that for every target speed visual presentation (multimodal combination MMC1) generates the largest error, while all the other multimodal combinations (MMC2-MMC4) reduce the tracking error. The benefit of acoustic modes as compared to pure graphics mode as a function of speed is shown in Figure 4.

Benefit of adding acoustic modes is in a range between $16 \%$ (for the lowest speed), and 6\% for the highest speed. It is worth noting that optimal multimodal combination varies with speed. Multimodal combination of visual and both acoustic modes (MMC4) gives highest benefit for the lowest target speed. With increased target speed, multimodal combination of graphical and acoustic position mode (MMC2) is optimal. For the highest target speed the best results are generated using acoustic distance mode as a supplement to visual presentation (MMC3).

Paired t-tests between multimodal combinations with audio modes and pure graphics mode for the same speed revealed significant differences in average error across five tests marked with '*' on Figure 4.

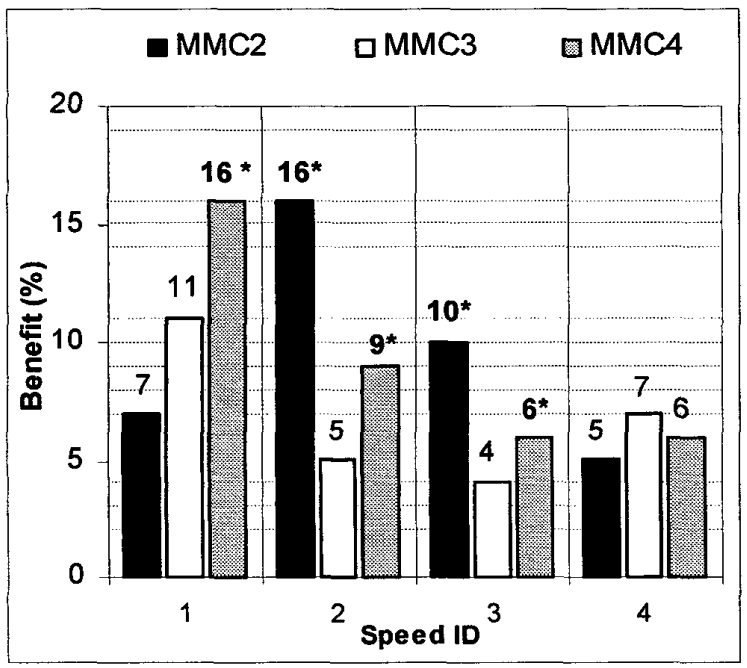

Figure 4. Performance improvement of multimodal presentation; values marked with '*' are statistically significant.

In first speed only MMC4 showed statistically significant benefit ( $\mathrm{p}=0.03)$. In second speed statistically significant benefits were found for MMC2 $(p=0.01)$ and MMC4 $(p=0.03)$. In third speed MMC2 $(p=0.001)$ and MMC4 $(p=0.003)$ again have shown statistically significant benefits. In the forth speed none of the multimodal combinations with sound showed statistically significant benefit.

\section{CONCLUSION}

Tactical audio display, as a tactical aid to achieve precise positional control in augmented reality systems, exhibits significant potential for improved user performance and better quality human-computer interface. We concluded that open multimodal environment, that we used to develop our system, facilitates very efficient rapid application development. Despite relatively poor audio quality of standard PC systems the experiment in our environment has shown that usage of acoustic modes improves human-machine interaction.

User customized settings, similar to user desktop settings, are also very important for tactical audio systems due to individual perceptual preferences. Although the experiment has shown that the average results are better with multimodal presentation, few participants did not exhibit performance improvement.

We plan to extend our experiment with three-dimensional acoustic guidance and additional situation critical information, such as prioritized set of threats. Future research will also include larger set of experiments, analysis of user learning curves, and multimodal guidance of truly portable, PDA based, guidance system.

\section{REFERENCES}

[1] Meera M. Blattner, "Multimodal Integration", IEEE Multimedia, Winter 1996, pp. 14-24;

[2] Sharon Oviatt, "Ten Myths of Multimodal Interaction", Comm. of the ACM, Vol. 42, No 11, Nov 1999, pp. 74-81;

[3] Begault DR, 3D Sound for Virtual Reality and Multimedia, Academic Press, Inc., Boston, 1994;

[4] Kramer, G., Ed., Auditory Display, Sonification, Audification and Auditory Interfaces, Addison Wesley, 1994.

[5] E. Jovanov, D. Starcevic, V. Radivojevic, A. Samardzic, V. Simeunovic, "Perceptualization of biomedical data", IEEE Engineering in Medicine and Biology Magazine, Vol. 18, No. 1, pp. 50-55, 1999;

[6] E. Jovanov, K. Wagner, V. Radivojevic, D. Starcevic, M. Quinn, D. Karron, "Tactical Audio and Acoustic Rendering in Biomedical Applications", IEEE Transactions on Information Technology in Biomedicine, Vol. 3, No. 2, June 1999, pp. 109-118;

[7] Wegner K, Karron D, "Surgical Navigation Using Audio Feedback." In Morgan KS et al (eds): Medicine Meets Virtual Reality: Global Healthcare Grid. IOS Press, Ohmsha, Washington, D.C., pp 450-458, 1997;

[8] E. Jovanov, Z. Obrenovic, D. Starcevic, D.B. Karron, "A Virtual Reality Training System for Tactical Audio Applications", SouthEastern Simulation Conference SESC'99, Huntsville, Alabama, Oct 1999, pp. 149-154;

[9] H. Sowizral, K. Rushforth, M. Deering, The Java 3D API Specification, Second Edition, Addison-Wesley Pub Co, Januar 2000, ISBN: 0201710412 